\title{
L-DOPA, BIPERIDENO E EXCREÇĀO SEBÁCEA NA DOENÇA DE PARKINSON
}

\author{
JOÃO C. B. VILLARES *
}

\begin{abstract}
RESUMO - A excreção sebácea frontal de 47 parkinsonianos «de novo» antes e após tratamento com anticolinérgico (biperideno), levodopa + IDAA e bromocriptina foi avaliada peIo método do ácido ósmico. Outros 100 parkinsonianos sob terapéutica crônica com biperideno, levodopa + IDAA ou associaçāo de ambos foram avaliados. Parkinsonianos «de novo» do sexo masculino apresentam valores de excreção sebácea significativamente mais elevados em relacão às mulheres. Verificou-se que biperideno nāo foi eficaz em reduzir c grau de excreção sebácea. Já, em relaçáo a L-dopa + IDAA constatou-se que a droga foi efetiva em reduzir - grau de excreção sebácea (NC e TRE) tanto no sexo masculino quanto no feminino. Em relação à bromocriptina (10mg/dia) tambêm constatou-se que houve redução da excreşão scbácea no sexo masculino. Correiacão significante positiva foi verificada entre o NC, tremor, bradicinesia, hipertonia, alteraçōes da marcha e postura e incapacidade funcional, entre parkinsonianos do sexo masculino e faixa etária 50-59 anos, no período pré-tratamento. Após o periodo de tratamento não mais havia correlação entre excreçăo sebácea e as manifestaçōes neurológicas da doença de Parkinson. Entre parkinsonianos sob terapêtica crônica verificou-se correlação positiva e significante entre excreção sebácea e bradicinesia. o grau de excreção sebácea de parkinsonianos «de novo» sem tratamento não difere do grau daqueles sob tratamento crônico, exceção feita a parkinsonianos com idade $\geqslant 60$ anos, em que verificou-se maior grau de excreção sebácea (NC e TRE) em relação ao mesmo sexo e faixa etária, sem tratamento. L-dopa + IDAA foi eficiente em reduzir o grau de excrecão sebácea de parkinsonianos «de novo», tornando-a significativamente menor em relação àqueles sob tratamento crônico. Não há diferença entre o grau de excreção sebácea de parkinsonianos «de novo» sem tratamento e parkinsonianos sob associação de drogas cronicamente. A possibilidade de ser a disfunção dopaminérgica hipotalâmica a origem do aumento da excreção sebácea em parkinsonianos é discutida, além de serem comparados os resultados apresentados aos registrados na literatura.
\end{abstract}

\section{Effects of L-dona and biperiden on the sebum secretion in Parkinson's disease.}

SUMMARY - Sebum secretion was measured on the forehead of 47 patients with idiopathic Parkinson's disease before and after treatment with anticholinergic (biperiden), levodopa + AAID and bromocriptine, by the osmic acid technique. Another 100 patients under biperiden, levodopa + AAID or association of both, for at least one year, were also evaluated. The male parkinsonian «de novo» patients have shown greater sebum secretion than female patients. It was also concluded that biperiden failed to reduce sebum secretion rate. On the other hand, it was found that L-dopa + AAID reduces the sebum secretion (CL $=$ casual level and $\mathrm{SER}=$ sebum excretion rate) on both male and female patients. Bromocriptine (1Gmg/day) was the second dopaminergic therapy employed in the present work. Similarly to L-dopa, this dopaminergic agonjst was able to significantly reduce sebum secretion (both CL and SER) of male patients. There was a positive and significant correlation for the 50-59 years old male patients «de novo» between CL and tremor, hypokinesia, gait and posture or functional incapacity, before treatment. After a period of treatment correlation

* Departamento de Psicobiologia, Escola Paulista de Medicina. Este trabalho, financiado por FAPESP, FINEP, CNPq e AFIP, é parte das exigências para a obtenção do título de Doutor em Ciências, por J.C.B.V., junto à Escola Paulista de Medicina. 
was no more found. In relation to parkinsonians under chronic treatment was found $\mathbf{a}$ positive and significant correlation between sebum secretion and hypokinesia. The level of sebum secretion on parkinsonian «de novo» patients before treatment was equal to parkinsonian patients under chronic treatment regardless the treatment, except for $\geqslant 60$ years old parkinsonians who have shown CL and SER higher than «de novo» parkinsonian patients with the same age but without treatment. The treatment with L-dopa + AAID significantly decreased both CL and SER of «de novo» parkinsonian patients. No difference of sebum secretion was found between parkinsonian «de novo» patients before treatment and patients under treatment, regardless the drugs, but after treatment with L-dopa. + AAID, «de novo» patients have shown significant lesser sebum secretion than patients under the same treatment for at least one year. It is discussed whether the suppressive effect of the dopaminergic drugs may be explained through dopamile hypothalamic dysfunction.

O aumento da excreção sebácea é fenômeno descrito na sindrome parkinsoniana pós-encefalite 11,19, por uso de neurolépticos 5,6 e, também, na doença de Parkinson idiopática 20, imputando-se o déficit dopaminérgico hipotalâmico como fator fundamental, muito embora seja ainda desconhecida a razâo pela qual esse fato ocorre. Drogás que reduzem a excreção de prolactina, por ação sobre os receptores dopaminérgicos D2, além da melhora do quadro clínico 21 , reduziriam a excreção sebácea 2,27 de parkinsonianos. Todavia, não somente drogas dopaminérgicas apresentariam atividade sobre a excreção sebácea, pois fenômeno semelhante toi também descrito para anticolinérgicos em parkinsonianos 7.

Embora a excreção sebácea seja aspecto clinico bem conhecido em parkinsonianos, há até o presente escasso conhecimento sobre a influência da levodopa e de anticolinérgicos sobre esta disfunçăo vegetativa. Assim sendo, o propósito desta investigação é avaliar os efeitos de drogas antiparkinsonianas tanto sobre a excreção sebácea quanto sobre a sintomatologia neurológica de parkinsonianos idiopáticos 'de novo', bem como avaliar os mesmos parâmetros em parkinsonianos sob utilização crônica de drogas, compará-los entre si e correlacionar suas manifestaçōes neurológicas à excreção sebácea.

\section{MATERIAL E METODOS}

Pacientes com diagnóstico de doença de Parkinson idiopática (DP): clois grupos experimentais foram constituidos (Tabelas 1 e 2). No grupo I foram alocados parkinsonianos já sob terapia crônica, classificando-os de acorủo com a droga em uso: 1. biperideno (anticolinérgico; 11 homens e 14 mulheres); 2. levodopa + benserazide (L-dopa † IDAA; 9 homens e 16 mulheres); 3 , associaçāo biperideno mais L-dopa + IDAA (30 homens e 20 mulheres). Nesses subgrupos, os diferentes pacientes erum incluidos, se já estivessem recebendo pelo menos $6 \mathrm{mg}$ de biperideno ao dia e/ou $500 \mathrm{mg}$ de L-dopa + IDAA, há pelo menos um ano. 0 grupo II se refere ao conjunto de parkinsonianos «de novo» participantes de um estudo prospectivo do efeito de drogas sobre a excreção sebácea e sintomatologia neurológica. Assim, alocaram-se os pacientes, de acordo com a droga fornecida, em: 1. pacientes que receberam somente biperideno; 2. pacientes que receberam L-dopa + IDAA; 3 . pacientes que receberam somente bromocrintina $(10 \mathrm{mg} / \mathrm{dia}-\mathrm{dose}$ fixa). Desta forma, 53 pacientes com o diagnóstico de DP idiopática «de novo» iniciaram o experimento preposto, muito embora somente 47 tenham vindo a completar us 127 dias de tratamento. Assim sendo, 15 pacientes (7 masculinos e 8 femininos) receberam biperideno na dose mínima de $6 \mathrm{mg} / \mathrm{dia}$; três pacientes do sexo feminino abandonaram o experimento. A outrus 21 pacientes (13 homens e 8 mulheres) foi estabelecida terapêtica com L-dopa + IDAA em doses variáveis de acordo $\mathrm{ccm}$ a necessidade clinica ât cada paciente. Finalmente, 17 pacientes (11 masculinos e 6 femininos) receberam 10mg/dia de bromocriptina: três pacientes do sexo feminino nāo completaram o experimento.

As manifestaçōes neurológicas da DP foram quantificadas pela escala da Universidade de New York (22), sendo incluídos no estudo somente parkinsonianos com escore global entre 20-60\%. Pacientes pertencentes ao grupo I foram avaliados uma única vez, enquanto as avaliaçōes daqueles do grupo II se deram antes do tratamento e após 7, 37 e 127 dias do inicio. Um só neurologista avaliou cada paciente. Este projeto foi previamente aprovado pelo Comitẽ de Etica e Pesquisa Clínica «in Anima Nobili» da Escola Paulista de Medicina. 


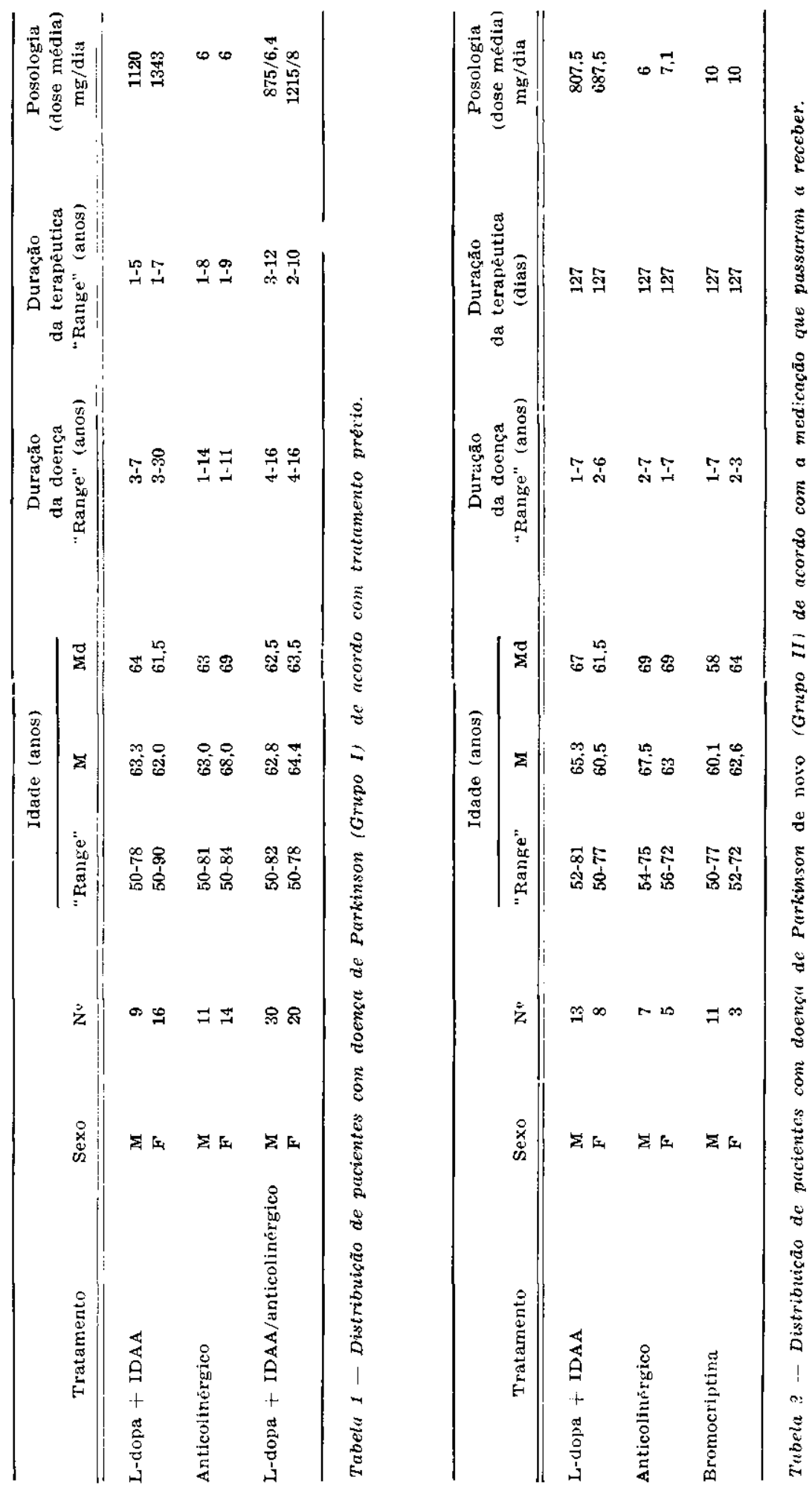


Técnica nara coleta do «sebum» - Inicialmente duas peças de papel Whatmann $n^{\circ} 1$ para cromatografia, medindo $2,5 \times 2,5 \mathrm{~cm}$, previamente lavadas com éter e secas à temperatura ambiente (para remoçāo de eventual material lipidico nelas existente), eram aplicadas à regiāo frontal cefálica de cada probando, em decúbito dorsal, bilateralmente, procurando-se as posiçóes equijidistantes a $1,5 \mathrm{~cm}$ do plano sagital, passando pela linha média e a $1,0 \mathrm{~cm}$ acima das sobrancelhas. O contacto do papel foi de três minutos mantendo-se uma pressão constante de $500 \mathrm{~g}$ com saco de pano contendo arroz. Findo este tempo, os papéis eram retirados e procedia-se à limpeza da pele da regiảo frontal com solução álcool-éter $50 \%$. Decorridos 10 minutos a partir da limpeza, procedia-se nova avaliação da excreçāo sebácea, também por três minutos, com dois novos papéis de filtro. Através deste procedimento obtinham-se, portanto, duas avaliaçōes da excreçāo sebácea. A primeira foi chamada nível casual (NC) e a segunda, taxa de reposição da excreção sebácea (TRE) em 13 minutos ( 1 ). A seguir as peças de papel utilizadas eram rapidamente mergulhadas em solução de ácido ósmico a $1 \%$ e secas à temperatura ambiente, com o que os lípides presentes ficavam evidenciados sob a forma de manchas pretas permanentes. Este procedimento baseou-se em modificaçōes da técnica colorimétrica de impregnaçāo do papel contendo lípides (1.3), uma das formas clássicas para a avaliação da excreçāo sebácea.

Escala para građuação da excreção sebácea - A escala de graduação da excreção sebácea em escores variáveis de 0 a 9 pontos ou graus, foi elaborada com 4 voluntários, limpando-se a pele da região frontal deles e colocando-se os papéis de filtro a diferentes intervalos dt tempo após a limpeza $10,15,30,60,90,120,150,180$ e 240 minutos). Os papéis eram mantidos em contato com a pele da regjão frontal por exatamente três minutos. Após a coloração com a solução de ácido ósmico, o papel com maior concentração de material lipidico, entre os colocados ao 0 minuto üpós a limpeza (dos quatro voluntários), recebeu o grau 1; c papel com maior densidade lipidica entre aqueles expostos 15 minutos após a limpeza da regiāo frontal recebeu o grau $2 \mathrm{e}$, assim, sucessivamente. $O$ grau zero foi atribuído a um papel não exposto. Desta forma, elaborou-se uma escala ordinal, semi-quantitativa (Fig. 1). A leitura do material lipídico obtido de parkinsonianos baseou-se em comparações contra a escala descrita.

ESCALA PARA AVALJACAOA DE SEBORRË]A

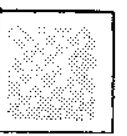

0

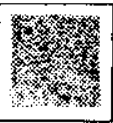

5

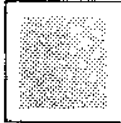

1

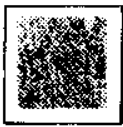

6

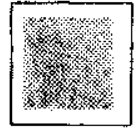

2

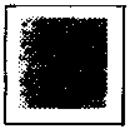

7

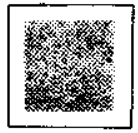

3

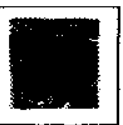

8

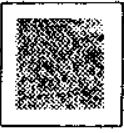

4

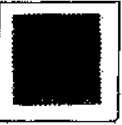

9
Fig. 1 - Escula ordinal, semiquantitativa, de avalią̧ão da excreção sebácea.

Estatística - A análise estatística constituiu-se na aplicação do teste de MannWhitney (25) para a relação entre os dois sexos. A análise de variância de Friedman (25), complementada pelo teste de comparaçôs múltiplas (15), foi aplicada para as amostras dependentes. Para as amostras independentes utilizou-se a análise de variância de KruskalWallis (26), complementada pelo teste de Dunn (16). O nivel de rejeiçào da hipótese de nulidade fo: tomado como igual ou menor at 0,05 ou $5 \%$.

\section{RESULTADOS}

Efeito de drogas sobre a excrecão sebácea de parkinsonianos «de novo» - Na figura 2 são mostrados os resultados. Verifica-se que, no periodo pré-tratamento, os pacientes du sexo masculino apresentam valores de excreção sebácea (NC e TRE) significativamente mais elevados en relação ảs mulheres ( $p \leqslant 0,05$; teste de Mann-Whitney). O tratamento com 
biperideno nāo modificou a taxa de excreção sebácea após 127 dias, quer em relaçāo ao sexo masculino quer em relação ao sexo feminino. Já em relação ao tratamento com L-dopa + IDAA, houve redução significante $(\mathrm{p} \leqslant 0,05 ;$ análise de variância de Friedman) da excreção sebácea (NC e TRE) tanto em relação ao sexo masculino quanto em relaçāo ao sexo feminino. Esta significância, em relaçāo ao sexo masculino, foi verificada (teste de comparaçōes múltiplas) entre o estado inicial (sem tratamento) e o $127 \%$ dia de tratamento $(p \leqslant 0,01)$ para o NC e entre o estado inicial e o 7॰, $37 \circ$ e $127 \circ$ dia de tratamento (p $\leqslant 0,05$ ) em relação a TRE sebácea. Quanto ao sexo feminino, houve diferença significante entre o estado inicial sem tratamento e $0127^{\circ}$ dia $(p \leqslant 0,01)$ após o início da droga, tanto em relaçảo ao NC quanto em relaçāo à TRE sebácea (Fig. 2).
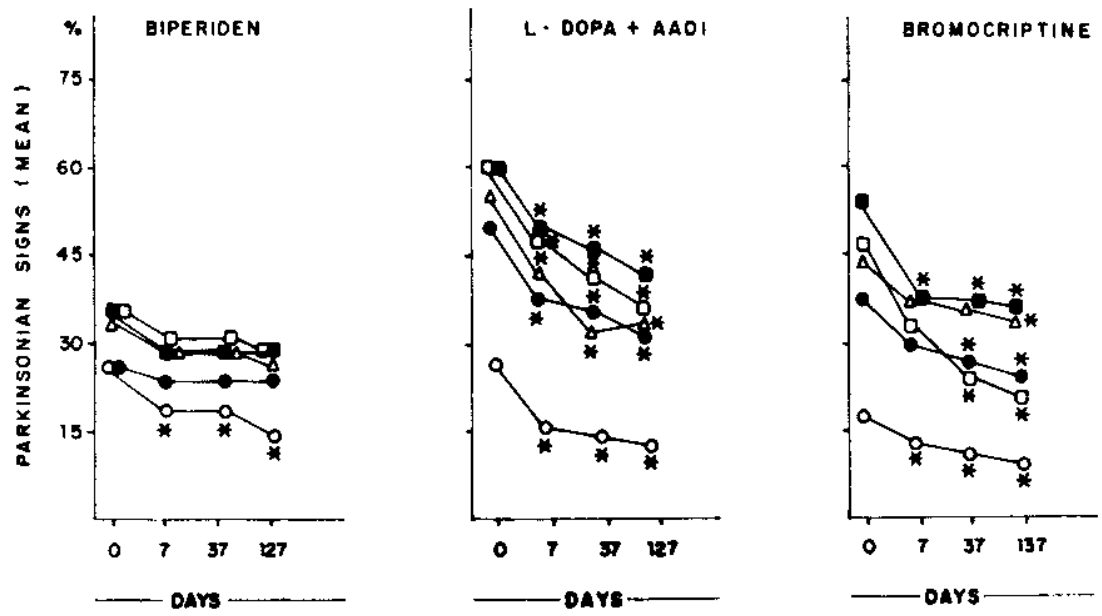

Fig. 2 - Efeito de droyas sobre a excreção sebácea de parkinsonianos 'de novo'.

Finalmente, na figura 2 é demonstrado que a bromocriptina também foi eficaz em reduzir $\circ$ NC e TRE de modo significante ( $\leqslant \leqslant 0,01$; análise de variância de Friedman) entre os parkinsonianos. Esta redução dá-se a partir do $7^{\circ}$ dia em relação ao $\left.\mathrm{NC}^{(p} \leqslant \mathbf{0 , 0 5}\right)$ e $37^{\circ}$ em relação à $T R E(p \leqslant 0,05$; teste de comparações múltiplas). Como somente três pacientes do sexo feminino completaram o periodo de tratamento, os dados não são suficientes para a análise estatística.

Correlaçāo entre excreçāo sebácea e manifestaçōes neurológicas da doença de Parkinson, em pacientes «de novo» (Grupo II), antes e após tratamento - Correlação positiva sígnificante foi verificada entre NC e o tremor, e a bradicinesia, e as alteracões da marcha $e$ postura e a incapacidade funcional ( $p \leqslant 0,05$; Spearman) entre pacientes do sexo masculino e faixa etária 50-59 anos, no periodo pré-tratamento. Nāo se constatou correlação significante entre a taxa de excreçāo sebácea e as manifestaçōes neurológicas em pacientes do sexo masculino com idade $\geqslant 60$ anos. Não se constatou qualquer correlação entre excreçāo sebácea e manifestaçôes neurológicas em relaçâo ao sexo feminino. Após o período de tratamento, verificuu-se que não mais ocorria correlaçāo significante entre a excrecão sebácea e as manifestaçōes neurológicas da doença de Parkinson. Esta constataçāo se deu entre os parkinsonianos tratados com đrogas dopaminérgicas (L-dopa + IDAA e bromocriptina) considerados como um só grupo.

Correlaçāo entre excreção sebácea e manifestaçōes neurológicas da doença de Parkinson em pacientes sob tratamento crônico - o grau de excreçāo sebácea, de forma geral, não se correlacionou com o grau das manifestacoóes neurológicas parkinsonianas, independentemente do tratamento a que os pacientes estavam submetidos (biperideno, L-dopa + IDAA ou biperideno e L-dopa + IDAA). Todavia, correlaçāo positiva e significante foi observada entre excreçao sebácea e bradicinesia ( $p \leqslant 0,05$; teste de Spearman).

Comparação entre grau de excreção sebácea de parkinsonianos «de novo» e crônicos $O$ grau de excreção sebácea de parkinsonianos «de novo» sem tratamento e após 127 dias 
sub biperideno nãc difere do daqueles sob tratamento crônico, independentemente do sexo e faixa etária. Por outro lado, parkinsonianos «de novo» sem tratamento, do sexo masculino, e parkinsonianos do mesmo sexo sob L-dopa + IDAA cronicamente, nāo apresentam diferença entre o grau de excreçåo sebácea e qualquer faixa etária e condição experimental. A mesma constatação foi verificada em relação ao sexo feminino para a faixa etária $50-59$ anos. Entretanto, ainda em relação ao sexo feminino, observou-se que parkinsonianos com idade $\geqslant 60$ anos, sob terapêutica crônica, apresentam maior grau de excreção sebácea, diferindo significantemente $(p \leqslant 0,05)$, tanto em relação ao NC quanto em relação à taxa de reposiçāo de excrecão sebácea daquelas parkinsonianas sem tratamento. Já o grau de excreçāo sebácea de parkinsonianos «de novo», há 127 dias sob terapêtica com L-dopa †. IDAA, é menor, diferindo significativamente daqueles parkinsonianos sob terapêutica crônica, quando se consideram o sexo masculino e as faixas etárias de 50-59 e $\geqslant 60$ anos, quer para o $\mathrm{NC}(p \leqslant 0,05)$ quer para a TRE sebácea $(p \leqslant 0,01)$. Em relação ao sexo femínino não se observou diferença para a faixa etária 50-59 anos, em qualquer condição experimental. Com relaçāo à faixa etária $\geqslant 60$ anos, parkinsonianas «de novo» apresentam menor grau de excreção sebácea, diferindo significantemente $(p \leqslant 0,05)$ em relação às parkinsonianas sob terapêutica crônica, em ambas condições experimentais. Não há diferença entre o grau de excreção sebácea de parkinsonianos «de novo» sem tratamento e parkinsonianos sob associação de L-dopa + IDAA e biperideno, qualquer que seja a condição experimental e a faixa etária.

\section{COMENTARIOS}

Disfunções autonômicas e endócrinas em pacientes com doença de Parkinson têm sido consideradas decorrentes de disfunçōes hipotalâmicas 17 . A razāo pela qual há aumento da excreção sebácea nos parkinsonianos pós-encefaliticos e também na doença de Parkinson idiopática ainda é desconhecida, mas há sugestão que responsabiliza o déficit de dopamina hipotálamo-hipofisária como fator primário 28.

Entre parkinsonianos 'de novo' que vieram a receber biperideno verificou-se que os do sexo masculino apresentam excreção sebácea (NC e TRE) mais elevada que os do sexo feminino, antes do início do tratamento. $O$ tratamento instituido não provocou a redução dos níveis de excreção sebácea ao longo dos 127 dias em ambos os sexos. Estes dados discordantes da literatura, estão também em desacordo com a açāo putativa da acetilcolina sobre a excreção sebácea exercida a nivel hipotalâmico, visto ter a aceitilcolina açāo facilitadora. O bloqueio da atividade colinérgica resultaria na redução dos niveis de excreção sebácea. Uma provável explicaçāo para esta verificaçāo, relaciona-se ao fato de que o déficit dopamınérgico nestes pacientes não era tão pronunciado, ao verificarmos os aspectos neurológicos.

Ao compararmos parkinsonianos 'de novo' após 127 dias de uso de biperideno e parkinsonianos sob terapêutica há mais de um ano, nāo verificamos diferenças significantes entre os graus de excreção sebácea. Também em relação aos parkinsonianos 'de novo' que receberam L-dopa + IDAA, verificou-se em relação ao sexo esse mesmo fato, ou seja, maior excreçăo sebácea (NC e/ou TRE) para o sexo masculino, em relação ao sexo feminino. A terapêutica com L-dopa + IDAA mostrou-se efetiva em reduzir o grau de excreção sebácea, tanto em relação ao sexo masculino quanto em relação ao sexo feminino, seja no NC ou TRE sebácea. Verificou-se também que parkinsonianos podem não apresentar excreção sebácea aumentada e que o efeito da L-dopa + IDAA não se produz em todos os casos nos quais há excreção sebácea elevada.

Ao compararmos parkinsonianos 'de novo' do sexo masculino e feminino (exceto $\geqslant 60$ anos) sem tratamento e parkinsonianos sob L-dopa + IDAA cronicamente, constatamos que não havia diferença entre o grau de excreção sebácea em relação ao sexo e faixa etária. O tratamento de parkinsonianos 'de novo' foi eficaz em reduzir a excreção sebácea a ponto de verificarmos que o grau de excreção sebácea tornou-se menor, diferindo significativamente daqueles que estavam sob L-dopa + IDAA cronicamente. Também entre aqueles parkinsonianos 'de novo', que vieram a receber bromocriptina, constatou-se que os homens apresentavam maior excreção sebácea, diferindo significativamente das mulheres. A ação deste agonista dopaminérgico foi efetiva em reduzir a excreção sebácea (NC e TRE) de parkinsonianos do sexo masculino. Confirmam-se portanto, as observaçöes de Streifler et al.27 e Agnoli et al.2. Em relação ao sexo feminino a terapêutica não foi efetiva, provavelmente, em decorrência do pequeno número de avaliados. Ao que parece, muito enbora as drogas dopaminérgicas provoquem redução do grau de excreção sebácea na fase inicial do tratamento, a longo prazo seu efeito deixa de existir, pois verificamos que parkinso- 
nianos 'de novo' sem tratamento e sob efeito crônico de drogas (L-dopa + IDAA, biperideno) não aprescentam diferenças entre os graus de excreção sebácea.

Burton et al. 8 concluiram que L-dopa é eficiente em reduzir a excreção sebácea de parkinsonianos, desde que ela estivesse presente. Os resultados obtidos por Harvilli e Appenzeller 15 também são efetivos em demonstrar que L-dopa reduz a excreção sebácea em parkinsonianos. Todavia, resultados opostos foram verificados por Cotterill et al.12. A confirmação de que L-dopa realmente reduz a excreção sebácea de parkinsonianos foi verificada por Appenzeller e Harvill 3 , concluindo que a redução da excreção sebácea precede a melhora do quadro neurológico. Muito embora a reduçāo da excreção sebácea também tenha sido constatada por Kohn et al.18, houve considerável variação nas respostas individuais, não podendo ser relacionadas com o grau de melhora do quadro clínico. Burton e Shuster 10 afirmam que há redução de $30 \%$ nos niveis de excreção sebácea (variação 17-42\%). A redução dos niveis de excreção sebácea de parkinsonianos 'de novo', e a ausência da manutenção do efeito provocado pela L-dopa, constatado ao compararmos parkinsonianos 'de novo' sem tratamento àqueles sob terapêutica crônica, poderia ser explicada pelo fenômeno de tolerância.

Dados contraditórios têm sido apresentados pela literatura em relação à correlação entre a excreção sebácea e as manifestaçōes neurológicas da doença de Parkinson. Baas et al.4 verificaram correlação linear entre a taxa de excreção sebácea, bradicinesia e rigidez, mas não verificaram correlação com o tremor. Por outro lado, Streifler et al.27 não constataram correlação significante entre a taxa de excreção sebácea $\mathrm{e}$ as manifestações neurológicas parkinsonianas. Kohn et al.18 também não verificaram paralelismo absoluto entre o grau de supressão de excreção sebácea e a melhora do quadro neurológico após a utilização de L-dopa. Entre os nossos parkinsonianos 'de novo' do sexo masculino com idade de 50-59 anos, no período prévio ao tratamento, constatou-se correlação positiva significante entre tremor, bradicinesia, alterações da marcha e postura, incapacidade funcional e excreção sebácea (NC). Ao término das diferentes terapêuticas propostas, não mais constatou-se a correlação acima, mesmo quando consideraram-se como único grupo pacientes sob drogas dopaminérgicas (L-dopa + IDAA e bromocriptina). Sob terapêutica crônica constatou-se correlação entre excreção sebácea e bradicinesia.

Verifica-se, da análise exposta, que excreção sebácea e bradicinesia correlacionam-se de forma praticamente constante, refletindo talvez a deficiência de dopamina nos diferentes sistemas dopaminérgicos. Até o presente, é desconhecido o mecanismo pelo qual a L-dopa e bromocriptina exercem os efeitos supressivos da excreção sebácea. Ação supressora direta sobre a glândula sebácea parece não ocorrer, pois a L-dopa é incapaz de reduzir a elevada excreção sebácea de pacientes com acne ${ }^{9}$. Shuster et al.25 afirmaram que provavelmente o hormônio melanócito estimulante (MSH) seria o fator hipofisário responsável pelo aumento da excreção sebácea em parkinsonianos. O possivel fator sebotrófico estaria sob controle inibitório 8 . A hipótese, aventada por Shuster et al.25, de que o aumento da excreção sebácea em parkinsonianos era conseqüente à ação do $M S H$, e em particular do beta-MSH, e que níveis séricos do beta-MSH estariam elevados em parkinsonianos, porém não em todos, nāo recebeu comprovaçăo.

A sugestão de que a sequência de aminoácidos presentes no MSH constitui tão somente artefato 23,24 , formado a partir da beta-lipotrofina (beta-LPH) e gamalipotrofina (gama-LPH) veio a comprovar que a hipótese proposta por Shuster et al.25, não era verídica. Todavia, se de um lado havia a inconsistência da presença do beta-MSH como um polipeptídeo com ação sebotrófica, de outro verificou-se que a beta-lipotrofina 29 apresenta ação sebotrófica. Fato ainda mais relevante é que a beta-lipotrofina está sob controle hipotalâmico inibitório exercido pela dopamina 14 . Assim sendo, a hipótese inicial de Shuster et al.25, na qual o hipotálamo através da dopamina exerceria efeito inibitório sobre a excreçăo sebácea permanece na ordem do dia.

\section{REFERENCIAS}

1. Agache P, Blane D - Currents status in sebum knowledge. Int J Derm 21:304, 1982.

2. Agnoli A, Casacchia M, Carolei A, Meco G, Zamponi A, Riggieri S - Aspetti neuroendocrinologici del morbo di Parkinson: interesse all'effecto terapeutico di un inibitore della prolattina. Minerva Med 67:3477, 1976.

3. Appenzeller O, Harvilli D - Effect of L-dopa on seborrhea of parkinsonism. Lancet 2:311, 1970. 
4. Baas H, Fischer PA, Schneider E - Influence of levodopa on seborrhea faciei in parkinsonism. Internat Symposium on Parkinson's Disease. New York, June 9-12, $1985, \mathrm{pg} 79$.

5. Binder RL, Jonelis FJ - Seborrheic dermatitis in neuroleptic-induced parkinsonism. Arch Derm 119:473, 1983.

6. Binder RL, Jonelis FJ - Seborrheic dermatitis: a newly reported side effect of neuroleptics. J Clin Psychiat 45:125, 1984.

7. Birkmayer W, Riederer P - Treatment. In: Parkinson's Disease. Wien, Springer, 1980, pg 89.

8. Burton JL, Cartlidge M, Shuster M - Effect of L-dopa on the seborrhea of parkinsonism. Br J Derm 88:475, 1973.

9. Burton JL, Libman LJ, Ha!l R, Shuster S - Levodopa in acne vulgaris, Lancet 2:370, 1971.

10. Burton JL, Shuster S - Effect of L-dopa on seborrhea of parkinsonism. Lancet 2:19, 1970 .

11. Cohn $\mathrm{T}-$ Encephalitis ohne Lethargie während der Grippe-epidemie. Neurol Zbl $28: 260$, 1920.

12. Cotterill JA, Cunliffe WJ, Willianson B, Arrowsmith WA, Cook JB, Summer D Sebum-excretion rate and skin-surface lipid composition in Parkinsin's disease before and during therapy with levo-dopa. Lancet $1: 1271,1971$.

13. EnderIin $\mathbf{K}$, Brun $\mathbf{R}$ - Nouvelles expériences sur la secrétion sébacée. Dermatologica $108: 235,1954$.

14. Franceschi $\mathbf{M}$, Cecchetto $\mathbf{R}$, Panerai AE, Truci G, Smirne $\mathbf{S}$, Canal $\mathbf{N}-$ Plasma beta-endorphine and beta-lipotropin in patients with Parkinson's disease. Clin neuropharmacol 9:549, 1986.

15. Harvilli D, Appenzeller O - A new approach to the reduction of sebum secretion. Arch Derm 103:492, 1971.

16. Hoilandier M, Wolfe DA - In: Nonparametric Statistical Methods. John Wiley \& Sons, New York, 1973, pg 503.

17. Javoy-Agid F, Ruberg M, Pique L, Bertagna K, Taquet H, Studier JM, Cesseli NF, Epelbaum J - Blochemistry of the hypothalamus in Parkinson's disease. Neurology $34: 672,1984$.

18. Kohn SR, Pochi PE, Strauss JS, Sax DS, Feldman RG, Timberlake WH - Sebaceous gland secretion in Parkinson's disease during L-dopa treatment. J Invest Derm 60:134, 1973.

19. Krestin D - The seborrhoeic facies as a manifestation of post-encephalitic parkinsonism and allied disorders. Quart J Med 21:177, 1927.

20. Kvorning SA - Excretion of skin lipids in natients with Parkinson's syndrome. Acta Derm Venerol 22:201, 1952.

21. La] S, Della Vega CE, Sourkes TK, Friesen HG - Effect of apomorphine on growth hormone, prolaction, luteinizing hormone and follicle-stimulating hormone levels in human serum. J Clin Endocr 37:719, 1973.

22. Lieberman A, Dziatolowski M, Gopinathan G, Kupersmith M, Neophytides A, Korein J - Evaluation on Parkinson's disease. In: Goldstein M, Calne DB, Lieberman A, Thorner MO, eds - Ergot compounds and Brain Function: Neuroendrocrine and Neuropsychiatric Aspects. Raven Press, New York, 1980, pg 277.

23. Scott AP, Lowry PJ - Adrenocorticotrophic and melanocyte-stimulating peptides in the human pituitary, Blochem J 139:593, 1975.

24. Scott APB, Rees LH - Proceedings: melanocyte-stimulating hormone related peptides in human plasma. J Endocr 63:51P, 1979.

25. Shuster S, Thody AJ, Goolamali SK, Burton JL, Plummer N, Bates D - Melanocyte stimulating hormone and parkinsonism. Lancet $1: 463,1973$.

26. Siegel S - Nonparametric Statistic for the Behavioral Sciences, McGraw-Hill, New York, 1956, pg 312 .

27. Streifler M, Avrami E, Rabey JM - L-dopa s.nd the secretion of sebum in parkinsonian patients. Eur Neurol 19:43, 1980.

28. Thody AJ, Shuster $\mathbf{S} \rightarrow$ Control of sebum secretion by the posterior pituitary. Nature $237: 346,1972$.

29. Thody AJ, Shuster S - Sebotrophic activity of beta-lipotrophin. J Endocr 50:533, 1971 . 